\title{
Searching through synaesthetic colors
}

\author{
BRUNO LAENG \\ University of Oslo, Oslo, Norway
}

\begin{abstract}
Synaesthesia can be characterized by illusory colors being elicited automatically when one reads an alphanumeric symbol. These colors can affect attention; synaesthetes can show advantages in visual search of achromatic symbols that normally cause slow searches. However, some studies have failed to find these advantages, challenging the conclusion that synaesthetic colors influence attention in a manner similar to the influence of perceptual colors. In the present study, we investigated 2 synaesthetes who reported colors localized in space over alphanumeric symbols' shapes. The Euclidian distance in CIE xyY color space between two synaesthetic colors was computed for each specific visual search, so that the relationship between color distance (CD) and efficiency of search could be explored with simple regression analyses. Target-to-distractors color salience systematically predicted the speed of search, but the CD between a target or distractors and the physically presented achromatic color did not. When the synaesthetic colors of a target and distractors were nearly complementary, searches resembled popout performance with real colors. Control participants who performed searches for the same symbols (which were colored according to the synaesthetic colors) showed search functions very similar to those shown by the synaesthetes for the physically achromatic symbols.
\end{abstract}

The intrusion of color sensations systematically triggered by stimuli that are not colored per se (e.g., black-printed alphanumeric symbols) has been labeled grapheme-color synaesthesia (e.g., Rich, Bradshaw, \& Mattingley, 2005; Simner et al., 2005). This conscious phenomenon is characterized by the experience of illusory colors' being elicited automatically when one reads alphanumeric symbols. Specifically, a synaesthete may report seeing the letter A as having two colors: one being that of the ink (or pixels), which depends on physical wavelengths, and the other being an illusory color that only the synaesthete experiences. Different from the illusory experiences of color afterimages, which can be verified easily by any normal observer, synaesthetic experience is absolutely "private" and highly idiosyncratic. In fact, every one of us can report private conscious events that others cannot immediately experience or directly verify (e.g., an internal pain, a dream). Despite the privacy of each brain's consciousness, we generally understand and accept the veridicality of statements about mental events, which can generally be referred to as a presently shared event (e.g., "This rose is dark red"), as the state of things in the external world, or as a memory of a similar event, which may be private (e.g., "I have a headache"). Thus, the phenomenon of synaesthesia would seem to further complicate the problem of human consciousness by adding "extra qualia" to an already deep "explanatory gap" (Wager, 1999). As Anne Treisman (2005) put it, color qualia normally derive from the senses, but the synaesthetic qualia derive indirectly from the identity of the associated shape, "so, a single feature gives rise to two different qualia" (p. 248).

Of course, one might seriously question the truth of these highly private color experiences, which may be confused with metaphorical play and attempts to seek social attention. However, it has been shown that some synaesthetes can identify and localize a target object (e.g., one digit 5) among distracting objects (e.g., several digits 2) more efficiently than nonsynaesthetes can, if the target and distractors differ by a synaesthetic color feature (e.g., Laeng, Svartdal, \& Oelmann, 2004; Palmeri, Blake, Marois, Flanery, \& Whetsell, 2002). For some of these individuals, despite the display's being completely achromatic in physical terms, the synaesthetic colors can cause an accelerated narrowing of attention onto a purely synaesthetically defined odd-man-out element of a scene. This type of evidence strongly suggests that the synaesthetic colors behave similarly to physically present colors, so that when there is a perceptual difference between target and distractors, this will affect the efficiency of a visual search, speeding up the whole process of finding the target.

Palmeri et al. (2002) showed that the synaesthetic advantage in "difficult" or serial visual search occurred only 
when the synaesthetic colors of a target and distractors were widely different from each other. Laeng et al. (2004) replicated these results with achromatic alphanumeric stimuli and proposed that when a synaesthete searches for an item defined by a conjunction of shape features, an early attentional fixation might, by chance, include the target. Within this account, the synaesthetic advantage in visual search would not derive from the singleton synaesthetic color's attracting attention to itself automatically (according to some preattentive processing that bypasses symbol identification); instead, the attention window would shift serially, sampling a cluster of symbols in parallel (cf. Treisman, 1982; Treisman \& Gelade, 1980), so that the occasional inclusion of the target within its focus would, already at the stage of partial processing, cause a cascading pattern of activation that enhances the target's salience, resulting in an accelerated narrowing of the focus. Thus, over a large number of trials, there would be more efficient target identification, as compared with nonsynaesthetes' searches for the same targets without the benefit of the synaesthetic colors.

However, the findings above have been challenged by subsequent studies by Edquist, Rich, Brinkman, and Mattingley (2006) and Sagiv, Heer, and Robertson (2006; see also Sagiv \& Robertson, 2005), which found no significant advantages in visual search rates in groups of synaesthetes. As will be discussed in more detail below, these negative findings should not lead one to the general conclusion that the perception of synaesthetic colors plays no role in such tasks; they both indicate the existence of several constraints on the nature of synaesthetic perception (Sagiv et al., 2006). It will become clear from the present study that the synaesthetic benefit is a graded process and that advantages become appreciable only when the perceptual salience of targets versus distractors is high. Moreover, as suggested by the existing literature, it seems likely that advantages in visual search can be observed most clearly for those individuals whose synaesthetic phenomenology is characterized by the perception of colors as being localized in space, especially when the shape of the inducing symbol itself appears to be colored.

Thus, the goal of the present study is to investigate and provide further evidence for the existence of a synaesthetic benefit in visual search. If the efficiency of any particular search is dependent on the salience of a target among its distractors (Duncan \& Humphreys, 1989; Rosenholtz, 1999), one would expect searches to become progressively more efficient the larger the Euclidean distance within color space between the synaesthetic color of the target letter and the synaesthetic color of the distractors. This effect would be best captured by comparing slope coefficients of the regression lines between distractors' set size and response times (RTs). Such a result would confirm that the synaesthetic colors of both the target and the distractors participate in generating the synaesthetic advantage, instead of such a benefit simply arising either (1) from the early termination of a search upon the unique match between an item and the synaesthetic color of the target or (2) via the more efficient rejection of distractors within a series of attentional fixations (as originally proposed by Palmeri et al., 2002).
The Euclidean distances between colors can be computed easily by using the triplets of RGB color coordinates obtained from each synaesthete's selections with the color palette of Microsoft Word and then computing from them the corresponding CIE xyY values. Once the desired coordinates are obtained, it is a small step to apply the Euclidean distance formula for three dimensions. In other words, the greater the difference between the CIE xyY values of the synaesthetic color of the target and those of the distractors, the easier it should be for the synaesthete to spot the target; conversely, the more similar the color of the target to that of the distractors, the harder it should be to find the target. Note that no colors were physically presented to the participants in this experiment and that the above predictions were entirely based on the hypothesized differences in synaesthetic colors alone. Thus, 2 synaesthetes (P.M. and T.H.) and a group of control participants were asked to search on the computer screen for predefined targets among numerically variable sets of predefined distractors. However, it is also of interest to compare results from the achromatic searches with those of a group of nonsynaesthetes who searched among chromatic symbols. Therefore, the nonsynaesthetes also performed the same visual search tasks described above, the only difference being that targets and distractors appeared in colors that were congruent with those reported by the synaesthetes. If the virtual colors can influence attention in a manner similar to the way real colors can, the search functions - that is, the slopes of the regressions of RTs over color distance (CD) - with chromatic symbols for nonsynaesthetes should be similar to those with monochromatic symbols for synaesthetes.

\section{METHOD}

\section{Participants}

P.M., a 62-year-old female musician and music teacher, and T.H., a 58-year-old female psychologist, participated in this study. They both reported grapheme-color synaesthesia; for them, the letters of the alphabet and the 10 digits occupied various positions within color space (see Figure 1). The participants reported no synaesthesia for any other stimuli.

Both P.M. and T.H. had normal vision and color sensitivity. Color sensitivity was assessed with the Farnsworth-Munsell 100 Hue Test (P.M.'s score $=26$; T.H.'s score $=68$ ) and Ishihara pseudoisochromatic plates (P.M.'s error score $=0$; T.H.'s error score $=1$ ). P.M. and T.H. showed high consistency in pairing colors to symbols, as revealed by selecting the same colors from the Pantone palette of Microsoft Word 2000 in two sessions (several months apart) and

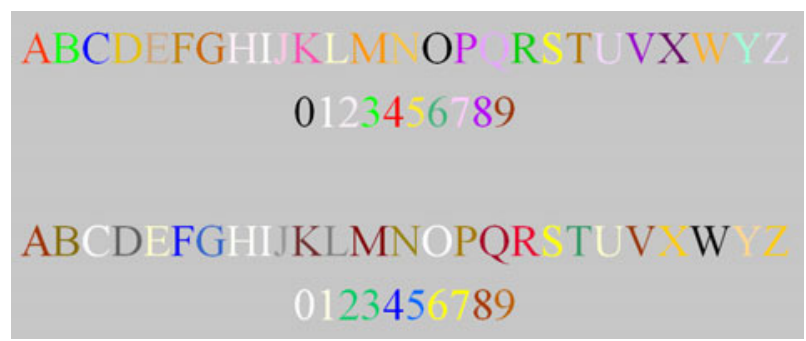

Figure 1. Alphanumeric symbols as colored in Microsoft Word 2000 by P.M. (top two rows) and T.H. (bottom two rows). This figure appears in full color in the online publication of this article. 
then correlating each session's values for each symbol and for each of three dimensions of color: hue, saturation, and luminance $(.83<$ $R<.99$ ). RGB coordinates were converted into their corresponding CIE xyY coordinates with the use of Bruce Lindbloom's CIE color calculator (www.brucelindbloom.com). The control participants $(N=12)$ were matched to P.M. and T.H. by age (mean $=57 ; S D=$ 4.6), sex, and educational level, and they performed the same tasks that were designed for the 2 synaesthetes.

\section{Stimuli and Procedure}

In each search task, there was one target to be found among a variable number of distractors (e.g., "Find the letter P among the Bs"). In all tasks, the distractors' set size varied from 1 to $20(N=640)$, and there was a $50 \%$ probability of the presence (in random positions) of the target. Table 1 lists the target and distractor pairs used in each task in their synaesthetic colors (as indicated by the synaesthetes' own coloring of the symbols). Note, however, that these colored stimuli appeared only for the visual searches performed by the control participants. In the searches performed by the synaesthetes, no symbol was colored; all of the stimuli were presented in standard black $(\mathrm{RGB}=0,0,0)$.

The task was self-paced, and the participants initiated each trial by pressing the spacebar on the computer keyboard. This caused a search array to appear immediately on the computer screen. Participants indicated the presence of a target by pressing the $\mathrm{Z}$ key (which was relabeled "Yes") or the absence of a target by pressing the M key (which was relabeled "No"). Pressing one of the response keys caused the array to disappear from the screen and be replaced by a blank screen. Stimulus presentations were controlled by SuperLab software, which also stored each keypress and RT.

\section{RESULTS}

Descriptive statistics were first calculated for each participant, obtaining mean RTs and mean percent accuracy rates for each combination of the variables (dis- tractor set size, target-present/target-absent). Accuracy was high (controls, $M=99 \%$; synaesthetes, $M=98 \%$ ); hence, errors were not submitted to inferential statistics analyses. Control participants' performance revealed that all searches were "difficult"; that is, slopes' coefficients of the search functions were all significantly different from 0 . In other words, there was no evidence of the target's "popping out" of the display. In addition, $t$ tests were computed by comparing a slope from 1 observer against a null hypothesis of 0: According to the formula by Howell (1987), $t=(b)\left(s_{x}\right)(\sqrt{N-1}) / s_{y-x}, d f=N-2$ (where $b=$ slope coefficient; $s_{x}=$ standard error of the regressor or number of distractors; $s_{y-x}=$ standard error of estimate, which is computed as $s_{y-x}=s_{y} \sqrt{1-r^{2}}$, with $s_{y}=$ standard error of the dependent variable or RTs and $r=$ Pearson's correlation coefficient). It was found that the slope coefficients did not differ significantly from 0 (or from popout performance) in the following searches: P.M.'s searches for $2 \mathrm{~s}$ among $5 \mathrm{~s}$ and for Bs among Ps; T.H.'s search for As among 4s; and the control participants' chromatic searches for the same symbols. Table 1 shows the slope coefficients for target-present trials and the corresponding $t$ tests of significance levels.

Synaesthetes' searches were progressively more efficient the larger the Euclidean distance between the synaesthetic colors of the target letter and those of the distractors. Figure 2 plots the searches' slope coefficients over the CD, as expressed in CIE xyY differences. The regression function, $Y=15.4-1.8 e^{-4} * X$, had a highly significant slope $[t(7)=5.6, p=.001]$ and a high correlation $\left(R^{2}=.84\right)$. Interestingly, when $\mathrm{CD}$ was large (e.g., P.M.'s searches for $2 \mathrm{~s}$ among $5 \mathrm{~s}$ ), the search's slope coefficient did not differ

Table 1

Target and Distractor Symbols, Color Distances (CD), Mean Response Times (RTs, in Milliseconds), Slope Coefficients of Search Function, $t$ Values, and $p$ Values

\begin{tabular}{|c|c|c|c|c|c|c|c|c|}
\hline \multirow[b]{2}{*}{ Participant(s) } & \multirow[b]{2}{*}{ Target } & \multirow[b]{2}{*}{ Distractor } & \multirow[b]{2}{*}{$\mathrm{CD}$} & \multicolumn{2}{|c|}{ RT } & \multirow[b]{2}{*}{ Slope } & \multirow[b]{2}{*}{$t$} & \multirow[b]{2}{*}{$p$} \\
\hline & & & & $M$ & $S D$ & & & \\
\hline P.M. & A & 4 & 3,573 & 796 & 148 & 14.5 & 8.9 & .0001 \\
\hline T.H. & A & 4 & 29,500 & 852 & 246 & 7.98 & 1.0 & .12 \\
\hline Controls & A & 4 & 0 & 832 & 198 & 16.7 & 10.2 & .0001 \\
\hline P.M. & $\mathrm{A}(255,52,2)$ & $4(254,0,0)$ & 3,573 & 778 & 102 & 13.0 & 7.4 & .0001 \\
\hline T.H. & A $(153,51,0)$ & $4(1,0,252)$ & 29,500 & 539 & 36 & 2.5 & 1.9 & .02 \\
\hline P.M. & 5 & 2 & 48,217 & 698 & 84 & 3.8 & 0.7 & .23 \\
\hline T.H. & 5 & 2 & 35,252 & 819 & 248 & 10.8 & 2.5 & .01 \\
\hline Controls & 5 & 2 & 0 & 912 & 112 & 18.4 & 8.1 & .0001 \\
\hline P.M. & $5(252,233,43)$ & $(248,238,246)$ & 48,217 & 577 & 33 & 2.6 & 0.7 & .23 \\
\hline T.H. & $5(0,103,254)$ & $2(0,204,101)$ & 35,252 & 664 & 55 & 6.4 & 1.7 & .06 \\
\hline P.M. & $\mathrm{B}$ & $\mathrm{P}$ & 76,869 & 684 & 46 & 2.1 & 0.8 & .21 \\
\hline T.H. & B & $\mathrm{P}$ & 13,858 & 936 & 286 & 14.2 & 7.8 & .0001 \\
\hline Controls & B & $\mathrm{P}$ & 0 & 888 & 152 & 12.8 & 7.5 & .0001 \\
\hline P.M. & $\mathrm{B}(2,253,0)$ & P $(197,15,240)$ & 76,869 & 565 & 40 & 2.3 & 0.7 & .23 \\
\hline T.H. & В $(133,109,3)$ & $\mathrm{P}(163,121,0)$ & 13,858 & 843 & 163 & 17.2 & 8.2 & .0001 \\
\hline P.M. & $\mathrm{M}$ & $\mathrm{N}$ & 5,690 & 762 & 101 & 13.5 & 7.9 & .0001 \\
\hline T.H. & M & $\mathrm{N}$ & 20,770 & 775 & 174 & 12.0 & 7.2 & .0001 \\
\hline Controls & $\mathrm{M}$ & $\mathrm{N}$ & 0 & 723 & 98 & 16.7 & 9.1 & .0001 \\
\hline P.M. & M $(253,152,0)$ & $N(246,200,62)$ & 5,690 & 699 & 70 & 13.1 & 7.3 & .0001 \\
\hline T.H. & $\mathrm{M}(128,0,1)$ & N $(150,129,4)$ & 20,770 & 646 & 78 & 10.1 & 6.8 & .0003 \\
\hline
\end{tabular}

Note-The $t$ tests were computed by comparing a slope coefficient against a null hypothesis of zero slope (Howell, 1987). Nonsignificant values indicate that the slope coefficient was not different from a flat search function over number of distractors (i.e., from the null hypothesis of slope $=0$ ). All symbols were presented in black in the search experiments with the synaesthetes, as well as in the colors (RGB values in parentheses) shown in the experiments with the nonsynaesthetes (controls). 


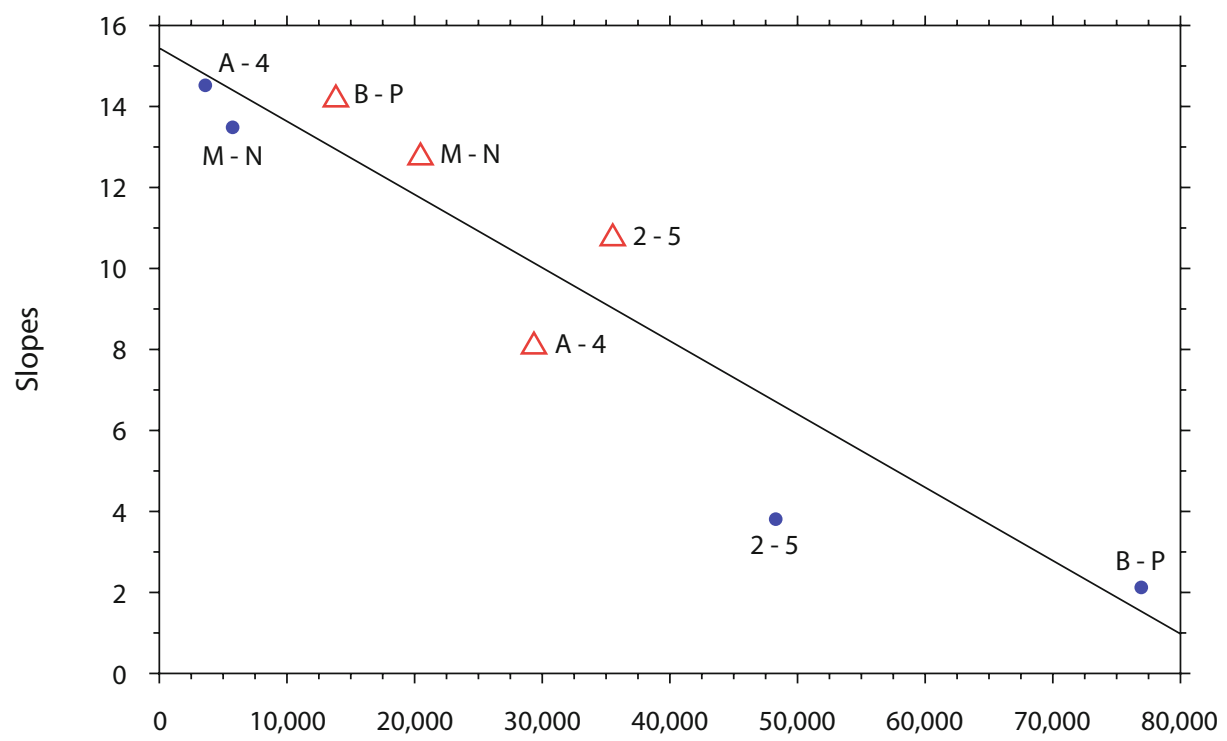

Figure 2. Regression of color distances (CDs) in CIE xyY coordinates between target and distractors and slope coefficients of T.H.'s (triangles) and P.M.'s (circles) search functions. The alphanumeric symbols indicate the task (first letter/digit $=$ target; second letter/digit $=$ distractor).

from 0 , and the search function was nearly flat and indistinguishable from that of a popout search (see also Laeng et al., 2004). As Figure 2 illustrates, even though the 2 synaesthetes searched through exactly the same symbol arrays, search efficiency was very different in each case. Efficiency clearly depended on the similarity between the synaesthetic colors evoked by each particular combination of symbols.

In addition, we computed distances of the targets' synaesthetic colors from the physical color of both the targets and the distractors-for example, standard black $(\mathrm{CIE}$ xyY $=0.345669,0.358496,0.0)$ - and we used this as a predictor for the search slopes in a simple regression analysis. Previous studies have indicated that the synaesthetic color of the distractors may be just as important as the synaesthetic color of the target for advantages in visual search; Sagiv et al. (2006) showed that when the distractors do not induce a synaesthetic color (so that they can be clustered together by color and facilitate guided searches; cf. Wolfe, 1994), the efficiency of search is greatly reduced or eliminated. Consistent with the account above, the analysis of the distance between the target's synaesthetic CD from the physical color of the distractors led to no significant relationship $[b=-.05 ; t(7)=0.9$, $p=.32]$. The analysis of the distance between the distractors' synaesthetic color and the physical color of the target also failed to reveal a significant relationship $[b=-.06$; $t(7)=1.4, p=.10$ ]. Clearly, it is not possible to predict the synaesthetes' behavior in visual search on the basis of the perception of only one synaesthetic color.

Finally, the control group of nonsynaesthetes performed the same visual search tasks a second time, the only difference being that, this time, the targets and distractors appeared in colors congruent (respectively) with those reported by the synaesthetes. When the searches' slope coef- ficients (averaged among the participants) were regressed over the CIE xyY CD differences, the regression function, $Y=13.2-1.9 e^{-4} * X$, also had a highly significant slope $[t(7)=2.9, p=.03]$ and a high correlation $\left(R^{2}=.59\right)$ (see Figure 3$)$. In addition, confidence intervals around the mean slope of searches of control participants with chromatic symbols were computed. It was found that search slopes for 5 s among 2 s that were shown as physically achromatic symbols differed significantly for both synaesthetes (for P.M., slope = 3.8; for T.H., slope = 10.8) from search slopes of the control participants' searches with the same symbols shown in color (confidence intervals for P.M.'s colors: upper $=3.4$, lower $=1.8$; confidence intervals for T.H.'s colors: upper $=7.8$, lower $=5.1$ ), since the former slopes' coefficients fell outside the confidence intervals of the latter. In other words, slopes were steeper for these synaesthetic searches than for the chromatic searches simulating the synaesthetic colors.

Finally, separate $t$ tests were used to compare the mean RTs in each of the physically achromatic search tasks for synaesthetes and each of the respective chromatic and achromatic searches for nonsynaesthetes. All of the physically achromatic searches by synaesthetes were significantly slower than the chromatic searches by nonsynaesthetes $(d f=638 ; 2.5<t<49.4, .001<p<.005)$. In addition, the physically achromatic searches by synaesthetes were also slower than the achromatic searches of nonsynaesthetes $(d f=638 ; 1.9<t<7.0, .001<p<$ $.05)$, except when the salience of the synaesthetic colors was large, in which case the synaesthetes were significantly faster than the control participants - namely, for P.M. in the $5 \mathrm{~s}$ among $2 \mathrm{~s}$ and Bs among Ps searches and for T.H. in the $5 \mathrm{~s}$ among $2 \mathrm{~s}$ searches $(8.6<t<38.7$, $.001<p<.01)$. 


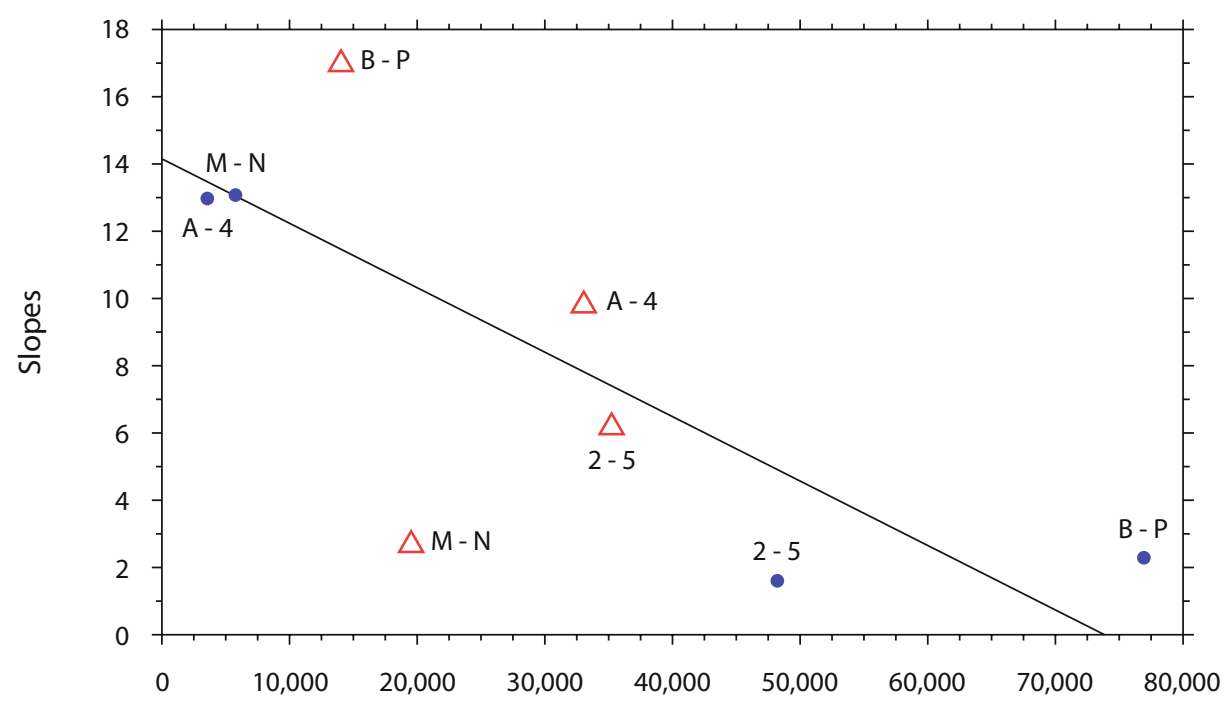

$C D$

\begin{abstract}
Figure 3. Regression of color distances (CDs) in CIE xyY coordinates between target and distractors and slope coefficients of the control participants' search functions when symbols were displayed in the same colors experienced (synaesthetically) by T.H. (triangles) and P.M. (circles). The alphanumeric symbols indicate the task (first letter/digit = target; second letter/digit $=$ distractor).
\end{abstract}

\section{DISCUSSION}

We examined how differences in the synaesthetic colors evoked by alphanumeric symbols could systematically affect the synaesthetes' ability to detect a target symbol among other distractor symbols. It is known from classic studies in the cognitive science of visual attention that the more salient a target's color is in relation to the color of the distractors in a crowded visual array (Duncan \& Humphreys, 1989; Treisman, 1991; Wolfe, 1994), the more efficient a search can be. When the perceptual difference is large, the target can pop out from the display.

The present findings show that the variability of search efficiency in synaesthesia can be systematically explained by the Euclidian distance within color space of the synaesthetic colors elicited by a particular pair of symbols. Indeed, according to our findings, these illusory colors' distances alone explained $84 \%$ of the variance in the efficiency of visual searches. There are at least two possible accounts of the present findings: (1) The synaesthete examines the symbols one at a time and can reject each distractor more rapidly when the apparent target-distractor color difference is larger, and (2) several items may be processed in parallel, so that the synaesthetic color of the target and that of the distractors are sometimes perceived simultaneously. In either account, the chromatic distance between the two synaesthetic colors would strongly predict the slope of the search function.

It was also found that there was no relationship between the search slope of the CD from either the target or the distractors and the physically presented achromatic color (black). The latter findings are consistent with those of Sagiv et al. (2006) - who also used 2 grapheme-color synaesthetes-where targets could induce synaesthetic colors, but none of the distractors induced synaesthetic colors. In their study, visual searches did not show any evidence that search rates for targets that induced a color were faster (or slopes less steep) than search rates for targets that did not induce a color. Palmeri et al. (2002) had also showed, by using distractors that did not induce colors, that the distractors' synaesthetic color is important for obtaining significant facilitative effects in visual search. Visual search studies of nonsynaesthetes have shown that the perceived similarity between colors displayed for the target and those displayed for the distractors predicts the time required to find the target (e.g., Reijnen, Wallach, Stöcklin, Kassuba, \& Opwis, 2007).

In addition, it was found that nonsynaesthetes' searches with colored symbols were, on average, faster than the synaesthetes' searches with the same (but achromatic) symbols. This finding supports previous conclusions (Laeng et al., 2004) that synaesthetic colors are not like the basic color features of a preattentive stage of processing. In fact, the facilitatory effects of synaesthetic colors might occur only after attention has been focused on the target (Sagiv et al., 2006). In other words, in these search tasks, the synaesthetes might scan around the array of symbols, and each attentional fixation would allow the synaesthetic colors to emerge, so that the added colors would "bootstrap" the narrowing of attention onto the target. This would cause an earlier response to targets of synaesthetes, as compared with those of nonsynaesthetes, since the synaesthetes would be able to convert the letter search into a color search (see Laeng et al., 2004). In contrast, in the physically chromatic searches, nonsynaesthete participants can already use the colors from the outsethence their faster responses in this condition, as compared with the synaesthetes' searches through synaesthetic col- 
ors. Most interestingly, P.M. was faster than the control participants in searching for a 5 among $2 \mathrm{~s}$ and a B among Ps, as was T.H. in searching for a 5 among 2 s. Notably, the colors of the 2 versus the $5 \mathrm{~s}$ and of the $\mathrm{B}$ versus the Ps fell close to the opposite ends of the color-opposition channels (i.e., blue-yellow and red-green; see Table 1) and would therefore be represented within a clear categorical subdivision of colors that is well known to result in efficient "guided" searches (Wolfe, 1994).

The present results appear to differ from those of a study by Edquist et al. (2006), who recruited 14 grapheme-color synaesthetes by use of a questionnaire, each of whom performed a visual search task in which a target digit was distinguished from surrounding distractors either by its unique synaesthetic color (achromatic search) or by its unique physical display color (chromatic search). Both synaesthetes and controls showed the expected efficient (popout) search slopes when the target was defined by a unique display color. In contrast, search slopes for both groups were equally inefficient when the targets and distractors were achromatic, despite eliciting distinct colors for the synaesthetes under normal viewing conditions. Thus, Edquist and colleagues were unable to reject the null hypothesis. Nevertheless, as they pointed out, one must be cautious in concluding for the absence of a difference, given also that several studies (Laeng et al., 2004; Palmeri et al., 2002; Smilek, Dixon, \& Merikle, 2003; the present study) have shown clear effects of synaesthetic colors on visual searches.

Notably, Edquist et al.'s (2006) study did not take into account CDs between targets and distractors, and, in fact, they paired each synaesthete's RTs in two searches, each with a different target (i.e., two different synaesthetic colors) among the same set of distractors. Thus, one cannot exclude that even large differences in CDs (e.g., blue vs. yellow) between one of the two targets and the distractors would be reduced by averaging them with those of the less salient target (e.g., blue vs. green). Considering the present study's findings and the hypothesis that salience between target and distractors systematically affects search rates, it is not too surprising that Edquist and colleagues were unable to reject the null hypothesis. As is also shown in the present study, not all searches may show significant gains in efficiency, since this depends on the target-todistractors salience in each specific search.

Finally, not every synaesthete may show perceptual-like effects on behavior of their synaesthetic colors. In fact, in Edquist et al.'s (2006) study, 4 synaesthetes did not report the synaesthetic colors as localized in space; only 6 out of the 14 synaesthetes strongly agreed with the phenomenological descriptions "the color is out in space" and "when I look at a letter or number I see a color"; only 3 agreed with "the color looks like it is on the page." Several researchers have pointed out that there may exist different forms of synaesthesia (i.e., of mechanisms producing the phenomenology), some of which may derive from strong semantic associations instead of being based on perceptual-like color sensations (e.g., Dixon, Smilek, Duffy, Zanna, \& Merikle, 2006; Dixon, Smilek, \& Merikle, 2004; Hubbard, Arman, Ramachandran, \& Boynton, 2005; Mar- tino \& Marks, 2001; Rich \& Mattingley, 2002; Ward, Li, Salih, \& Sagiv, 2007). In addition, there is evidence that the neural substrate of the perceptual (projectors) versus the semantic (associators) type of synaesthesia may be different from - that is, greater than - the normal volume of connective fibers among cortical areas of projectors, as compared with associators and nonsynaesthetes (Rouw \& Scholte, 2007). Thus, considering the fact that both P.M. and T.H. strongly reported synaesthetic colors as being localized or spread over the symbols (as compared with the heterogeneity in phenomenology of the participants in Edquist and colleagues' study), it is difficult to conclude that previous negative evidence challenges the conclusion that synaesthetic color differences can-like real perceptual colors can - modulate the efficiency of visual search.

The present findings indicate that synaesthetic colors can be triggered rapidly as a synaesthete searches through symbols for a target, accelerating the target's discovery. As indicated by the performance of the control group, these visual search tasks would normally require serially focused attentional shifts; nevertheless, the synaesthetic colors of the targets and their distractors have a strong influence on visual search, which can overcome the similarities in shape features between targets and distractors. The present findings are consistent with those showing the existence of early perceptual-like effects in the synaesthetic phenomenology.

\section{AUTHOR NOTE}

Correspondence concerning this article should be addressed to B. Laeng, Department of Psychology, University of Oslo, 1094 Blindern, 0317 Oslo, Norway (e-mail: bruno.laeng@psykologi.uio.no).

\section{REFERENCES}

Dixon, M. J., Smilek, D., Duffy, P. L., Zanna, M. P., \& Merikle, P. M. (2006). The role of meaning in grapheme-colour synaesthesia. Cortex, 42, 243-252.

Dixon, M. J., SmileK, D., \& Merikle, P. M. (2004). Not all synaesthetes are created equal: Projector versus associator synaesthetes. Cognitive, Affective, \& Behavioral Neuroscience, 4, 335-343.

Duncan, J., \& Humphreys, G. W. (1989). Visual search and stimulus similarity. Psychological Review, 96, 433-458.

Edquist, J., Rich, A. N., Brinkman, C., \& Mattingley, J. B. (2006) Do synaesthetic colours act as unique features in visual search? Cortex, 42, 222-231.

Howell, D. C. (1987). Statistical methods for psychology (2nd ed.). Boston: Duxbury.

Hubbard, E. M., Arman, A. C., Ramachandran, V. S., \& Boynton, G. M. (2005). Individual differences among grapheme-color synesthetes: Brain-behavior correlations. Neuron, 45, 975-985.

Laeng, B., Svartdal, F., \& Oelmann, H. (2004). Does color synesthesia pose a paradox for early-selection theories of attention? Psychological Science, 15, 277-281.

Martino, G., \& Marks, L. E. (2001). Synesthesia: Strong and weak. Current Directions in Psychological Science, 10, 61-65.

Palmeri, T. J., Blake, R., Marois, R., Flanery, M. A., \& WhetSELL, W., JR. (2002). The perceptual reality of synesthetic colors Proceedings of the National Academy of Sciences, 99, 4127-4131.

Reijnen, E., Wallach, D., Stöcklin, M., Kassuba, T., \& Opwis, K. (2007). Color similarity in visual search. Swiss Journal of Psychology, 66, 191-199.

Rich, A. N., Bradshaw, J. L., \& Mattingley, J. B. (2005). A systematic, large-scale study of synaesthesia: Implications for the role of early experience in lexical-colour associations. Cognition, 98, 53-84. 
Rich, A. N., \& Mattingley, J. B. (2002). Anomalous perception in synaesthesia: A cognitive neuroscience perspective. Nature Reviews Neuroscience, 3, 43-52

Rosenholtz, R. (1999). A simple saliency model predicts a number of motion popout phenomena. Vision Research, 39, 3157-3163.

Rouw, R., \& ScholTe, H. S. (2007). Increased structural connectivity in grapheme-color synesthesia. Nature Neuroscience, 10, 792-797.

Sagiv, N., Heer, J., \& Robertson, L. C. (2006). Does binding of synesthetic color to the evoking grapheme require attention? Cortex, $\mathbf{4 2}$, 232-242.

Sagiv, N., \& Robertson, L. C. (2005). Synesthesia and the binding problem. In L. C. Robertson \& N. Sagiv (Eds.), Synesthesia: Perspectives from cognitive neuroscience (pp. 90-107). New York: Oxford University Press.

Simner, J., Ward, J., Lanz, M., Jansari, A., Noonan, K., Glover, L., \& OAKLEY, D. A. (2005). Non-random associations of graphemes to colours in synaesthetic and non-synaesthetic populations. Cognitive Neuropsychology, 22, 1069-1085.

SmileK, D., Dixon, M. J., \& Merikle, P. M. (2003). Synaesthetic photisms guide attention. Brain \& Cognition, 53, 364-367.

Treisman, A. [M.] (1982). Perceptual grouping and attention in visual search for features and for objects. Journal of Experimental Psychology: Human Perception \& Performance, 8, 194-214.

Treisman, A. [M.] (1991). Search, similarity, and integration of features between and within dimensions. Journal of Experimental Psychology: Human Perception \& Performance, 17, 652-676.

Treisman, A. [M.] (2005). Synesthesia: Implications for attention, binding, and consciousness. In L. C. Robertson \& N. Sagiv (Eds.), Synesthesia: Perspectives from cognitive neuroscience (pp. 239-254). New York: Oxford University Press.

Treisman, A. M., \& Gelade, G. (1980). A feature integration theory of attention. Cognitive Psychology, 12, 97-136.

WAGER, A. (1999). The extra qualia problem: Synaesthesia and representationism. Philosophical Psychology, 12, 263-281.

WARD, J., LI, R., SALIH, S., \& SAgiv, N. (2007). Varieties of graphemecolor synaesthesia: A new theory of phenomenological and behavioural differences. Consciousness \& Cognition, 16, 913-931.

Wolfe, J. M. (1994). Guided Search 2.0: A revised model of visualsearch. Psychonomic Bulletin \& Review, 1, 202-238.

(Manuscript received June 13, 2008;

revision accepted for publication May 3, 2009.) 